\title{
Minimum Energy Operation of Petlyuk Distillation Columns - Nonsharp Product Specifications
}

\author{
Ivar J. Halvorsen ${ }^{\mathrm{a}}$ and Sigurd Skogestad ${ }^{\mathrm{b}}$ \\ ${ }^{a}$ SINTEF ICT, Applied Cybernetics, N-7465 Trondheim, Norway \\ ${ }^{b}$ Norwegian University of Science and Technology (NTNU), Department of Chemical En- \\ gineering, N-7491 Trondheim, Norway.
}

\begin{abstract}
The Petlyuk or fully thermally coupled distillation arrangement has the ability to separate a ternary feed into three pure products with an energy consumption $20-30 \%$ below conventional arrangements. The most interesting realisation is the dividing wall column (DWC). The Petlyuk arrangement has typically 5 degrees of freedom: Boilup rate, reflux rate, side-stream rate and split ratios above and below the dividing wall. In order to achieve the potential savings in practice, the column has to be carefully controlled.
\end{abstract}

Keywords: Underwood equations, Fully thermally coupled columns,

\section{Introduction}

The objective of this paper is to present the general minimum energy solution for nonsharp product splits for the integrated Petlyuk distillation column shown in Figure 1 , and to discuss important aspects of operation of such columns.

The configuration in Figure 1 has five degrees of freedom after the level control loops in the top and bottom are closed. These are the main column reflux $\left(L_{T}\right)$, boi$\operatorname{lup}\left(V_{B}\right)$ and the sidestream flow rate $(S)$ which are used for product composition control, plus the reflux and vapour flow in the prefractionator, represented by the split ratios $\left(R_{l}, R_{v}\right)$. The latter two degrees of freedom are here used for minimizing the energy requirement.

Several authors (Fidkowski and Krolikowski 1986, Glinos et. al. 1989, Carlberg and Westerberg 1989) have presented expressions for the minimum energy solution for sharp product splits, and have pointed out that the

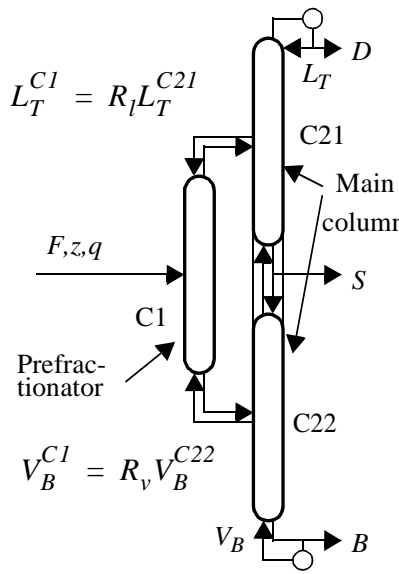

Figure 1: The integrated Petlyuk arrangement for separation of ternary mixtures 
a plane spanned by the two selected degrees of freedom. With optimal values of these two degrees of freedom, the energy requirement for the Petlyuk arrangement is typically $30 \%$ lower than in conventional column sequences.

It turns out that the inpurity specification for the sidestream product has a significant impact on the optimality region and thus on how the remaining two degrees of freedom should be used. We will show that the optimality region in the case of a nonsharp sidestream specification is extended from a line segment to a quadrangle-shaped region, with a width given by the sidestream purity only. The detailed deduction is given in the thesis by Halvorsen 2001, Chapter 9 .

Wolff and Skogestad 1995, Morud and Skogestad 1994 also discussed the operation of the Petlyuk arrangement when both the light and heavy sidestream impurity are specified. This may in fact lead to infeasible operation for certain selections of the split ratios.

\section{The Basic Methods}

The analysis and presentation is based on the $V_{\text {min }}$-diagram which is based directly on Underwood's equations for minimum energy for infinite number of stages (Underwood 1945-1948). Halvorsen and Skogestad 2003abc show how to apply these methods for minimum energy calculations for directly coupled arrangements. Here we give a brief review of the most important issues.

\subsection{The Underwood Equations}

The actual Underwood roots in the top $(\phi)$ and bottom $(\psi)$ of a two-product column are defined by the following relationships between the vapour flow $(V)$ and the net component flows ( $w$, defined positive upwards) through a cross-section in the top $(T)$ and in the bottom $(B)$ of the column. $\alpha_{i}$ is the relative volatility referred to the least volatile component.

$$
V_{T}=\sum \frac{\alpha_{i} w_{i, T}}{\alpha_{i}-\phi}, \quad \text { and } \quad V_{B}=\sum \frac{\alpha_{i} w_{i, B}}{\alpha_{i}-\psi}
$$

The common Underwood roots $(\theta)$, that characterize the minimum energy operation are obtained by solving the equation which arises when we subtract the equations above.

$$
\frac{V_{T}}{F}-\frac{V_{B}}{F}=\frac{\alpha_{A} z_{A}}{\alpha_{A}-\theta}+\frac{\alpha_{B} z_{B}}{\alpha_{B}-\theta}+\frac{\alpha_{C} z_{C}}{\alpha_{C}-\theta}=1-q
$$

Here the feed composition (z) appears since: $w_{i, T}-w_{i, B}=w_{i, F}=z_{i} F$. The minimum vapour flow when root $\mathrm{k}$ is active $\left(\phi_{k}=\theta_{k}=\psi_{k+1}\right)$, is then: 


$$
V_{\text {Tmin }}=\sum \frac{\alpha_{i} w_{i, T}}{\alpha_{i}-\theta_{k}}, \quad \text { and } \quad V_{B \min }=\sum \frac{\alpha_{i} w_{i, B}}{\alpha_{i}-\theta_{k}}
$$

\subsection{The $V_{\text {min }}$-Diagram}

Figure 2 illustrates a $V_{m i n}$-diagram for a given ternary feed mixture $(\mathrm{ABC})$ in a two-product column, e.g. for the prefractionator $(\mathrm{C} 1)$ in Figure 1. We use the top vapour flow $\left(\mathrm{V}_{\mathrm{T}}\right)$ and the net product split $(D / F)$ as degrees of freedom. The peaks represent minimum energy for sharp split between $\mathrm{A} / \mathrm{BC}\left(\mathrm{P}_{\mathrm{AB}}\right)$ or $\mathrm{AB} / \mathrm{C}$ $\left(\mathrm{P}_{\mathrm{BC}}\right)$. Sharp split between $\mathrm{A} / \mathrm{C}$ require operation above the $\mathrm{V}$-shaped $\mathrm{P}_{\mathrm{AB}}-\mathrm{P}_{\mathrm{AC}^{-}}$ $\mathrm{P}_{\mathrm{BC}}$, with minimum vapour flow at the preferred split $\left(\mathrm{P}_{\mathrm{AC}}\right)$. In the triangular regions under the "mountain", a set of components $\mathrm{AB}, \mathrm{ABC}$ or $\mathrm{BC}$ may be distributing to both products, and in each of these regions the active Underwood roots

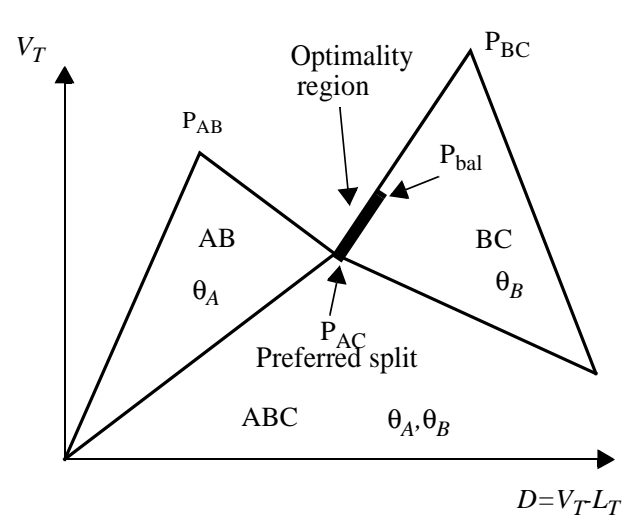

Figure 2: The $V_{\text {min }}$-diagram. The distributing components and the active Underwood roots are indicated in each region. will be the ones between the relative volatilities of the distributing products. Above the "mountain", $V>V_{\text {min }}$, only one component may distribute and there are no common Underwood roots.

\subsection{The Optimality Region for Sharp Product Splits}

Somewhat surprisingly, the minimum boilup solution for the arrangement is not unique, and the optimality region is the minimum boilup region in the space spanned by the two remaining degrees of freedom, here chosen as the net flow leaving the prefractionator and the vapour flow in the prefractionator $\left(D^{C 1}, V_{T}^{C 1}\right)$. As shown by Fidkowski and Krolikowski 1986, and revised in the $V_{m i n}$-diagram in Figure 2, the optimality region for the Petlyuk arrangement is when the prefractionator is operated along the line segment $\left(\mathrm{P}_{\mathrm{AC}^{-}}\right.$ $\left.\mathrm{P}_{\mathrm{bal}}\right)$. The extent is determined by different peaks, which indicates that minimum vapor flow is different in the top and in the bottom. Detailed derivation is found in Halvorsen and Skogestad 2003ab.

\section{Non-Sharp Product Specifications}

\subsection{Relation Between Compositions, Flows and Recoveries}

We choose to specify the products by the composition of the main component in each of the three product streams; at the top $(D)$, at the side $(S)$ and in the bottom $(B)$ $\left(x_{A, D}, x_{B, S}, x_{C, B}\right)$ (note that when $B$ is used in subscripts, the first position refer to com- 
ponent and the second to product or section). In normal operating regions, $\left(x_{C, D}=0\right)$, and $\left(x_{A, B}=0\right)$. In the sidestream, we may have both light $\left(x_{A, S}\right)$ and heavy $\left(x_{C, S}\right)$ impurities. Since $x_{A, S}+x_{B, S}+x_{C, S}=1$, we only need one of the sidestream impurities in addition to the three main specifications to determine the product streams uniquely. We here choose to use $x_{A, S}$ as a free variable. The overall material balance gives:

$$
\left[\begin{array}{c}
z_{A} \\
z_{B} \\
z_{C}
\end{array}\right] F=\left[\begin{array}{ccc}
x_{A, D} & x_{A, S} & 0 \\
\left(1-x_{A, D}\right) & x_{B, S} & \left(1-x_{C, B}\right) \\
0 & \left(1-x_{B, S}-x_{A, S}\right) & x_{C, B}
\end{array}\right]\left[\begin{array}{c}
D \\
S \\
B
\end{array}\right]=M_{S}\left[\begin{array}{c}
D \\
S \\
B
\end{array}\right]
$$

Observe that the product specification matrix $M_{S}=I$ for sharp product splits, and this gives a particular simple solution: $D=z_{A} F, S=z_{B} F, B=z_{C} F$.

For use of Underwood equations for directly coupled sections it is convenient to use net component flows $(w)$. These are found easily when the product flows and compositions are known:

$$
\begin{array}{lll}
w_{A, D}=x_{A, D} D & w_{A, S}=x_{A, S} S & w_{A, B}=0 \\
w_{B, D}=\left(1-x_{A, D}\right) D & w_{B, S}=x_{B, S} S & w_{B, B}=\left(1-x_{C, B}\right) B \\
w_{C, D}=0 & w_{C, S}=1-x_{B, S}-x_{A, S} & w_{C, B}=x_{C, B} B
\end{array}
$$

\subsection{Minimum Vapour Flow for Non-Sharp Product Specifications}

As shown in Halvorsen and Skogestad 2003bc for the 3-product Petlyuk column, and for the general M-product case, the minimum vapour flow for the Petlyuk column is the same as the maximum of the minimum energy required for any pair of product splits in a binary column. This is also valid for the nonsharp product splits between D/SB and DS/B.

$$
V_{T \min }^{P e t l}=\max \left(V_{T \min }^{D / S B}, V_{T \min }^{D S / B}\right)=\max \left(V_{T \min }^{C 21}, V_{B \min }^{C 22}+(1-q) F\right)
$$

Expressed by the Underwood roots:

$$
\begin{gathered}
V_{T m i n}^{D / S B}=\frac{\alpha_{A} w_{A, T}^{C 21}}{\alpha_{A}-\theta_{A}}+\frac{\alpha_{B} w_{B, T}^{C 12}}{\alpha_{B}-\theta_{A}}=V_{T m i n}^{C 21} \\
\left.V_{T \text { min }}^{D S / B}=\frac{\alpha_{B} w_{B, B}^{C 22}}{\alpha_{B}-\theta_{B}}+\frac{\alpha_{C} w_{C}^{C 22}}{\alpha_{C}-\theta_{B}}+(1-q) F\right)=V_{B \min }^{C 22}+(1-q) F
\end{gathered}
$$


However, since we only specify the main components in each of the three products, the impurity specification in the sidestream, here represented by $x_{A, S}$, is a remaining degree of freedom. Thus, in general the solution to (6) has to be minimized with respect to $x_{A, S}$ :

$$
V_{\text {Tmin }}^{\text {Petlyuk }}=\min _{x_{A, S}}\left(\max \left(V_{T \min }^{C 21}\left(x_{A, S}\right), V_{B \min }^{C 22}\left(x_{A, S}\right)+(1-q) F\right)\right)
$$

We illustrate the behaviour of the minimum energy operating points in Figure 3. Note that $V_{B \min }^{C 22}\left(x_{A, S}\right)$ is minimized for $x_{A, S}=0$ and $V_{T \min }^{C 21}\left(x_{A, S}\right)$ is minimized for $x_{A, S}=1-x_{B, S}$ which is the same as $x_{C, S}=0$.

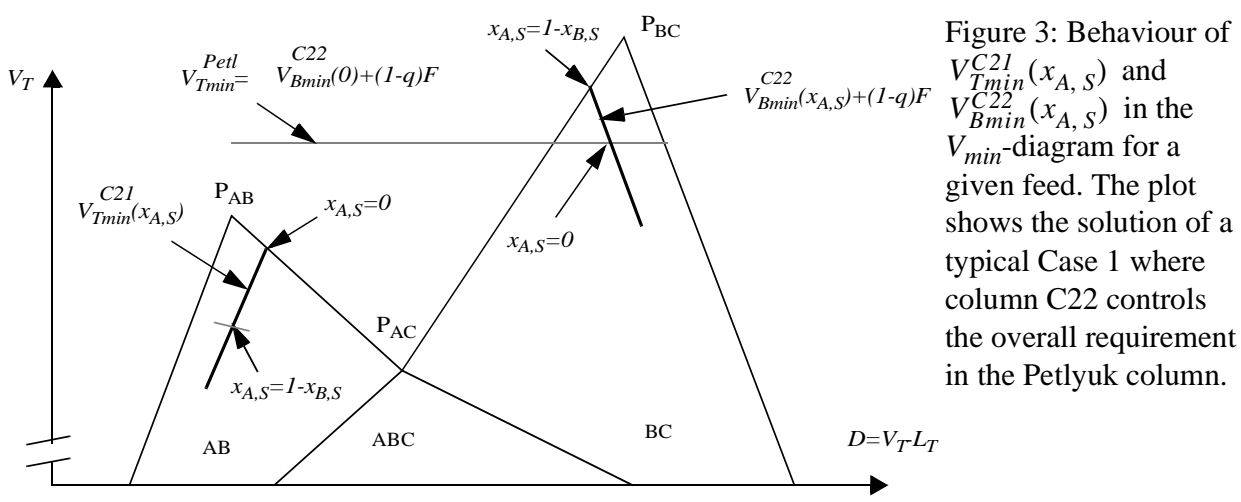

For the case in Figure 3, we obviously have $V_{B \min }^{C 22}(0)+(1-q) F>V_{T \text { min }}^{C 21}\left(1-x_{B, S}\right)$ This implies that the requirement in $\mathrm{C} 22$ controls the overall requirement, and the solution to (9) is found when $x_{A, S}=0$ and $V_{\text {Tmin }}^{\text {etlyuk }}=V_{B \min }^{C 22}(0)+(1-q) F$. We classify this as solution Case 1, and one characteristic is that we have only the heavy $\mathrm{C}$ component as impurity in the sidestream at the optimum.

Similarly, when the peak $\mathrm{P}_{\mathrm{AB}}$ is significantly higher than $\mathrm{P}_{\mathrm{BC}}$, we will have an optimal solution with only light A impurity in the sidestream (Case 3). We may only get a solution where the optimum is obtained for a combination of $\mathrm{A}$ and $\mathrm{C}$ impurity in the sidestream when the peaks are of similar height (Case 2).

These three cases are equivalent to similar cases for sharp product splits, and we summarize the characteristics of the possible solutions:

1. C22 controls: $V_{\text {Tmin }}^{P e t l}=V_{B \min }^{C 22}(0)+(1-q) F>V_{\text {Tmin }}^{C 21}\left(1-x_{B, S}\right)$ for

$$
x_{A, S}=O \text { and } x_{C, S}=1-x_{B, S}
$$


2. Balanced: $\quad V_{T \min }^{P e t l}=V_{B \min }^{C 22}\left(x_{A, S}\right)+(1-q) F=V_{T \min }^{C 21}\left(x_{A, S}\right)$ for

$$
0<x_{A, S}<1-x_{B, S} \text { and } x_{C, S}=1-x_{B, S}-x_{A, S}
$$

3. C21 controls: $V_{\text {Tmin }}^{\text {Petl }}=V_{\text {Tmin }}^{C 21}(0)>V_{B \min }^{C 22}\left(1-x_{B, S}\right)+(1-q) F$ for

$$
x_{A, S}=1-x_{B, S} \text { and } x_{C, S}=0
$$

\subsection{Do not reduce purity specification to save energy}

Note that the reduction in energy requirement when impurity is allowed in the products is almost linearly dependent of the impurity specifications, and that we do not obtain much energy saving by reducing the purity requirements.

Example: $V_{\text {Tmin }}^{\text {Petl }}=1.366$ for sharp product splits where the feed is given by: $\alpha=[4,2,1], z=[1 / 3,1 / 3,1 / 3]$ and $q=1$. For $99 \%$ sidestream purity in the sidestream, the requirement is reduced by just $1.0 \%$ to $V_{\text {Tmin }}^{\text {Petl }}=1.352$. Similarly, For $99 \%$ purity in all products, we obtain $1.6 \%$ reduction, to $V_{T \min }^{\text {Petl }}=1.343$. A feed with the given properties can e.g. be a mixture of Heptane, Octane and Nonane which has approximately these relative volatilities at atmospheric pressure.

Since energy savings in Petlyuk columns typically are in the range of 20-30\% compared to conventional arrangements, the further reduction due to impure product specifications will be insignificant and we will not recommend to use non-sharp specifications to save more energy. On the other hand, requirements to an optimal operation strategy is strongly affected by non-sharp product split specifications. This is because operation outside the optimality region may easily lead to losses comparable to the whole potential savings. High purity specification results in a narrow optimal operating region (the line segment), while low purity specification allow operation in a wider region (a quadrangle) which may be simpler to stay within. This is very important both when regarding the complexity of the column design and the required control system.

\subsection{The Optimality Region}

During operation of the column, the two remaining degrees of freedom (DOF) determine the actual operating point. The optimality region are all the possible operating points which results in minimum energy consumption as given by equation (9). For sharp product splits, the optimality region is the line segment PR in Figure 2. For nonsharp splits it is shown in Halvorsen 2001 that the line segment PR opens up to a quadrarangle as illustrated in Figure 4 . The width between the lines $P_{1}-R_{1}$ and $P_{2}-R_{2}$ is mainly determined by the sidestream impurity. The impurity in the top or sidestream has minor impact. The extent P-R is quite similar to the sharp-split case, and is mainly determined by the difference in vapour requirements in the top and bottom of the main column. 


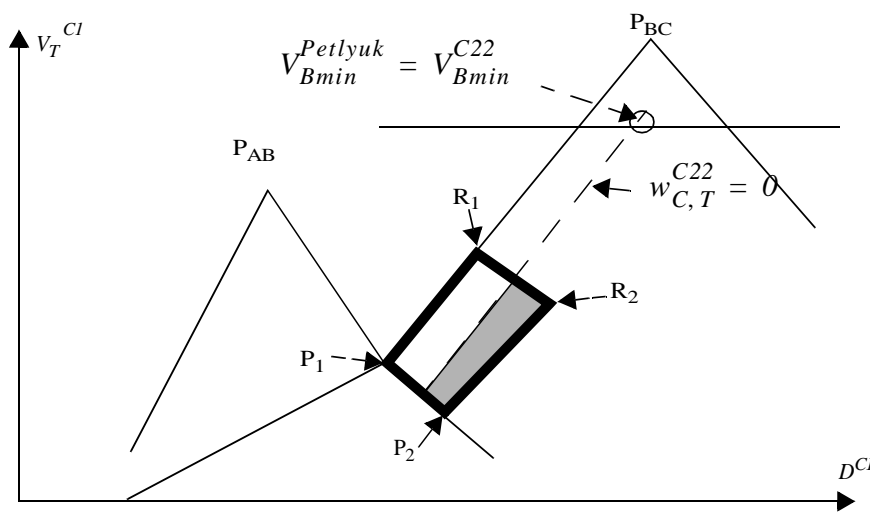

Figure 4: Optimality region for non-sharp side-product specification. The overall vapour flow in the Petlyuk arrangement is constant and optimal for the prefractionator column operated inside the optimality region (the bold quadrangle: $\left.\mathrm{P}_{1}-\mathrm{R}_{1}-\mathrm{R}_{2}-\mathrm{R}_{1}-\mathrm{P}_{1}\right)$.

\subsection{Optimality region and the complete solution surface}

We here introduce the split ratios as an alternative set of degrees of freedom: liquid split $R_{l}=L_{T}^{C 1} / L_{T}^{C 21}$ and vapour split $R_{v}=V_{B}^{C 1} / V_{B}^{C 22}$. We will now discuss the solution surface $V_{B}^{P e t l}\left(R_{l}, R_{v}\right)$ for nonsharp product splits, and infinite number of stages.

In Figure 5 a we show the optimality region for $97 \%$ purity in all three products. Feed data is $F=1, z=[1 / 3,1 / 3,1 / 3], \alpha=[4,2,1], q=0.5$, which corresponds to a mixture of Heptane, Octane and Nonane at atmospheric pressure. The total number of stages $N=440$, distributed in the individual sections as $N_{T}^{C 21}=N_{B}^{C 22}=30$ and $N_{B}^{C 1}=N_{T}^{C 1}=N_{B}^{C 21}=N_{T}^{C 22}=40$. This is in practice "infinite" number of stages for this separation task.
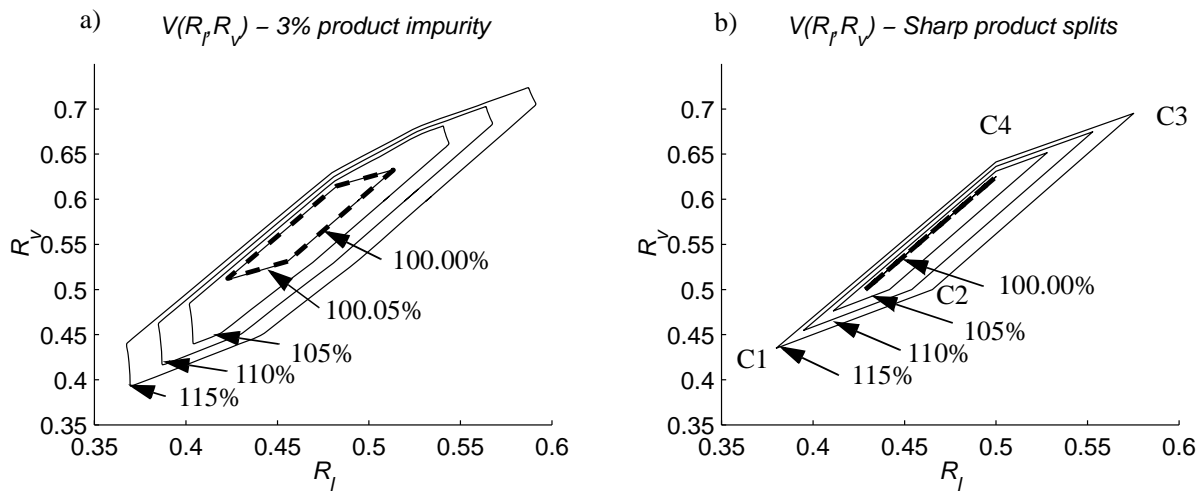

Figure 5: The whole solution surface $V\left(R_{l}, R_{v}\right)$ is widened for non-sharp products. The plots show contours of the solution surfaces for 3\% product impurity (a) and for sharp product splits (b) ( $V=100 \%$ in the optimality region). Note the characteristic sharp corners denoted C1-C4 on the sharp-split contours. 
Figure 5a shows contour plots for $V_{B}^{C 22}$ at $0.05 \%, 5 \% 10 \%$ and $15 \%$ above $V_{B \text { min }}^{P e t l}$. Observe that the optimality region computed for infinite number of stages (dashed) fits the $0.05 \%$ contour very well. This is a practical confirmation of the theoretical results.

We may compare with the corresponding sharp split case, shown in Figure 5b. The nonsharp solution surface is wider, not only at the optimality region, but at every contour of constant vapour flow. Thus for a given inaccuracy in implementation of the optimal degrees of freedom, it is more likely that there is a lower loss in the nonsharp case. However, the energy consumption increases rapidly outside the optimality region in both cases, so we still have to pay attention to setting the split ratios at proper values. We also clearly observe the same characteristic main "corners" for the non-sharp case, but not the corner "lines" has been "widened".

\section{Conclusions}

For high purity products the optimality region is a line segment in the plane spanned by the remaining two degrees of freedom when three have been used for product composition control. Non-sharp products bring new dimensions to the optimality region. In particular when we allow for an impure side-stream, optimal operation is achieved in a wider quadrangle-shaped region as opposed to the line segment. In this paper we present analytical expressions for minimum energy and the detailed boundaries of the optimality region for any purity specification. A practical consequence is that it is normally simpler to operate a Petlyuk column (or a DWC) with an impure side-stream. The result can also be used to diagnose operation where it seems difficult to obtain high side-stream purity and at the same time obtain the theoretical energy savings.

\section{References}

Carlberg, N.A. and Westerberg, A.W. (1989a). Temperature-Heat Diagrams for Complex. Columns. 3. Underwood's Method for the Petlyuk Configuration. Ind. Eng. Chem. Res. Vol. 28, p 1386-1397, 1989.

Fidkowski, Z. and Krolikowski, L. (1986). Thermally Coupled System of Distillation Columns: Optimization Procedure, AIChE Journal, Vol. 32, No. 4, 1986.

Halvorsen, I.J. Minimum Energy Requirements in Complex Distillation Arrangements. NTNU Dr. ing. Thesis, 2001:43.

Available from the web page of Sigurd Skogestad, Dept. of Chemical Engineering, NTNU (http://www.nt.ntnu.no/users/skoge/publications/thesis/2001_halvorsen/)

Halvorsen, I.J. and Skogestad S. Minimum Energy Consumption in Multicomponent Distillation. 1. $V_{m i n}$-Diagram for a Two-Product Column. IInd. Eng. Chem. Res. 2003, 42, 596-604,

Halvorsen, I.J. and Skogestad S. Minimum Energy Consumption in Multicomponent Distillation. 2. Three-Product Petlyuk Arrangements. Ind. Eng. Chem. Res. 2003, 42, 605-615, 
Halvorsen, I.J. and Skogestad S. Minimum Energy Consumption in Multicomponent Distillation. 3. More Than Three Products and Generalized Petlyuk Arrangements. IInd. Eng. Chem. Res. 2003, 42, 616-629,

Glinos, K.N. and Nikolaides, I.P. and Malone, M.F. (1986). New complex column arrangements for ideal distillation. Ind. Eng. Chem. Process Des. Dev, 1986, vol. 25, no 3, pp 694-699

Morud, J. and Skogestad, S. (1994). Allowable Operating Regions of Integrated Distillation Arrangements. AIChE Annual Meeting, San Francisco, USA, Nov. 13-18 1994, included in Morud (1995)

Underwood, A.J.V. et. al. (1945), Fractional Distillation of Ternary Mixtures. Part I. J. Inst. Petroleum, 31, 111-118, 1945

Underwood, A.J.V. et. al. (1946a), Fractional Distillation of Ternary Mixtures. Part II. J. Inst. Petroleum, 32, 598-613, 1946

Underwood, A.J.V. (1946b), Fractional Distillation of Multi-Component Mixtures - Calculation of Minimum reflux Ratio . Inst. Petroleum, 32, 614-626, 1946

Underwood, A.J.V. (1948), Fractional Distillation of Multi-Component Mixtures. Chemical Engineering Progress, Vol.44, no 8, 1948

Wolff, E. and Skogestad, S. (1995). Operation of Integrated Three-Product (Petlyuk) Distillation Columns, Ind. Eng. Chem. Res. 1995, 34, 2094-2103 
